\title{
PET findings after COVID-19 vaccination: “Keep Calm and Carry On”
}

\author{
Giorgio Treglia ${ }^{1,2,3,4,5} \cdot$ Marco Cuzzocrea ${ }^{1} \cdot$ Barbara Muoio $^{6} \cdot$ Luigia Elzi $^{6,7}$
}

Received: 22 March 2021 / Accepted: 6 May 2021 / Published online: 13 May 2021

(C) Italian Association of Nuclear Medicine and Molecular Imaging 2021

Large-scale worldwide vaccination programs against the 2019 coronavirus diseases (COVID-19) are being rapidly deployed. As this vaccination is becoming more widespread, we are observing an increase of patients with previous vaccination against COVID-19 who underwent ${ }^{18}$ F-FDG PET/ $\mathrm{CT}$ for different indications (i.e., cancer staging or restaging or evaluation of inflammatory diseases). Knowledge of vaccination-related effects is important to prevent wrong interpretations and alleviate patient concern during diagnostic imaging procedures. The earliest publications on this topic occurred in the field of breast imaging, where COVID19 vaccine-induced lymphadenopathy was cited as a cause of unilateral axillary lymphadenopathy [1].

Taking into account recent literature data, we are also observing a rapid increase of published scientific articles reporting PET findings with different radiotracers in patients with previous vaccination against COVID-19 [2-21, 27, 28].

Overall, these articles are mainly case reports or small case series recently published by research groups from different countries worldwide reporting PET findings in

Barbara Muoio and Luigia Elzi share the last authorship.

Giorgio Treglia

giorgio.treglia@eoc.ch

1 Clinic of Nuclear Medicine, Imaging Institute of Southern Switzerland, Ente Ospedaliero Cantonale, Bellinzona, Switzerland

2 Department of Nuclear Medicine and Molecular Imaging, Lausanne University Hospital, Lausanne, Switzerland

3 Health Technology Assessment Unit, Academic Education, Research and Innovation Area, General Directorate, Ente Ospedaliero Cantonale, Bellinzona, Switzerland

4 Faculty of Biology and Medicine, University of Lausanne, Lausanne, Switzerland

5 Faculty of Biomedical Sciences, Università della Svizzera Italiana, Lugano, Switzerland

6 Department of Medicine, Ente Ospedaliero Cantonale, Bellinzona, Switzerland

7 Faculty of Medicine, University of Basel, Basel, Switzerland
COVID-19 vaccine recipients who underwent PET/CT or PET/MRI with different radiotracers for several indications [2-21] (Table 1). Most of the described patients underwent vaccination against COVID-19 from 1 day to 3 weeks before ${ }^{18} \mathrm{~F}$-FDG PET/CT. About the ${ }^{18} \mathrm{~F}$-FDG PET findings after COVID-19 vaccination, most of the published articles reported increased radiophamaceutical uptake in axillary and subpectoral lymph nodes at the same side of the vaccine inoculation. Increased uptake in deltoid muscle corresponding to the vaccine inoculation site was also frequently described. Beyond the axilla, increased radiopharmaceutical uptake in supraclavicular and lower cervical lymph nodes was also illustrated in some reports. The hypermetabolic lymph nodes were normal-sized or enlarged. Less frequently, diffuse splenic ${ }^{18}$ F-FDG-uptake was also described. All these described sites of increased radiopharmaceutical uptake were interpreted as reactive due to immune response after recent vaccination against COVID-19 [2-10, 12-15, 17-21].

Radiopharmaceutical uptake in axillary lymph nodes was also described after PET/CT with radiolabelled choline or somatostatin analogues in COVID-19 vaccination recipients $[11,16]$.

We would like to underline that the main advantages of these case reports and small case series is to inform the nuclear medicine community about the increasing appearance of these PET findings following COVID-19 vaccination.

On the other hand, these findings are not surprising for the nuclear medicine physicians for several reasons $[22,23]$. First of all, it is well known that inflammatory cells may take up ${ }^{18} \mathrm{~F}$-FDG due to their increased glucose uptake and glycolytic activity. Therefore, ${ }^{18} \mathrm{~F}$-FDG is not a specific tracer for cancer cells and reactive lymph nodes may take up ${ }^{18} \mathrm{~F}$-FDG mimicking neoplastic lesions at PET. For these reason, ${ }^{18} \mathrm{~F}$ FDG PET/CT may also be used to evaluate inflammatory and infectious diseases with good diagnostic accuracy as demonstrated by several evidence-based manuscripts [24].

It is also not surprising that reactive lymph nodes may show increased ${ }^{18} \mathrm{~F}$-FDG uptake and normal size in some 


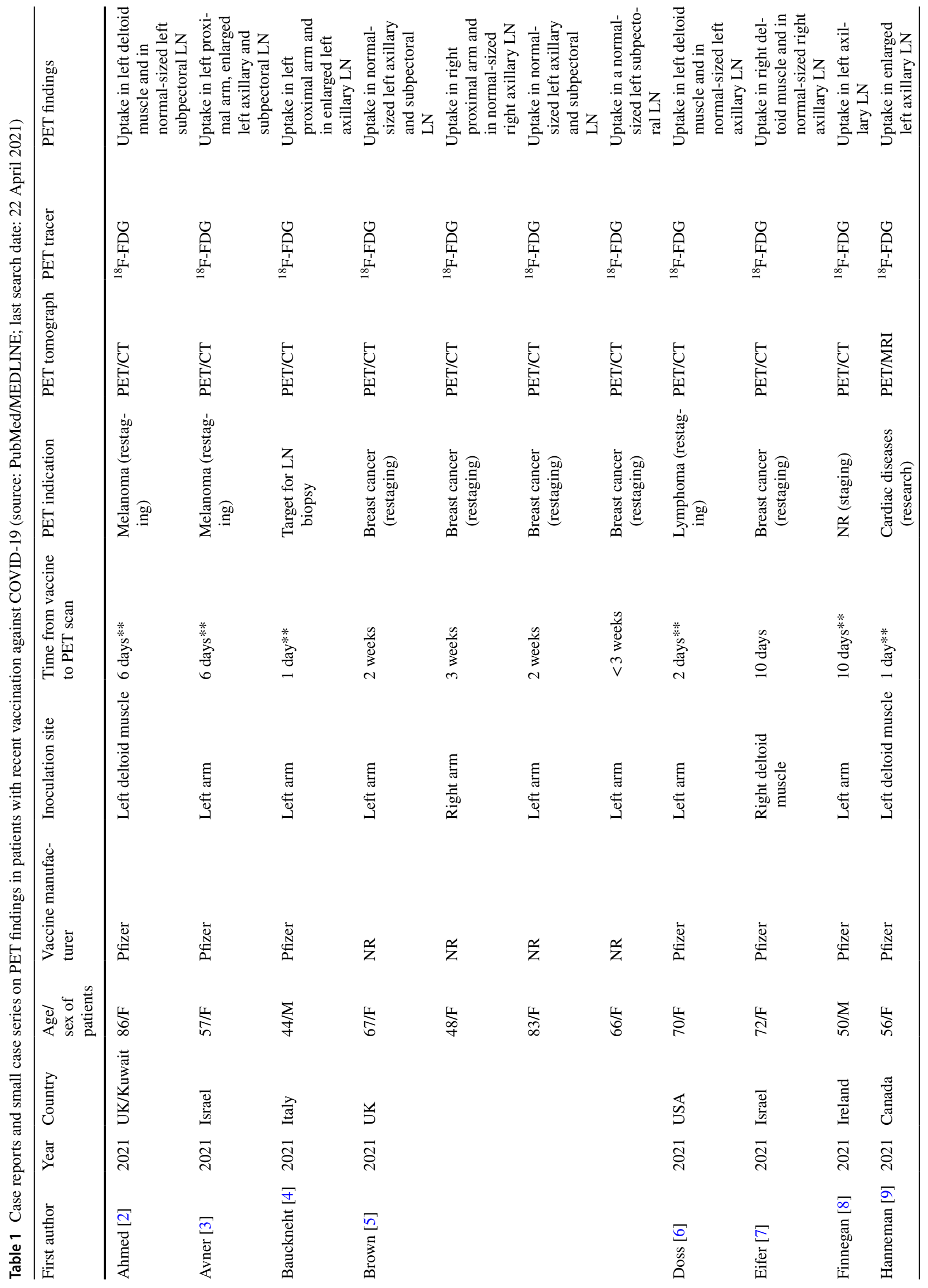




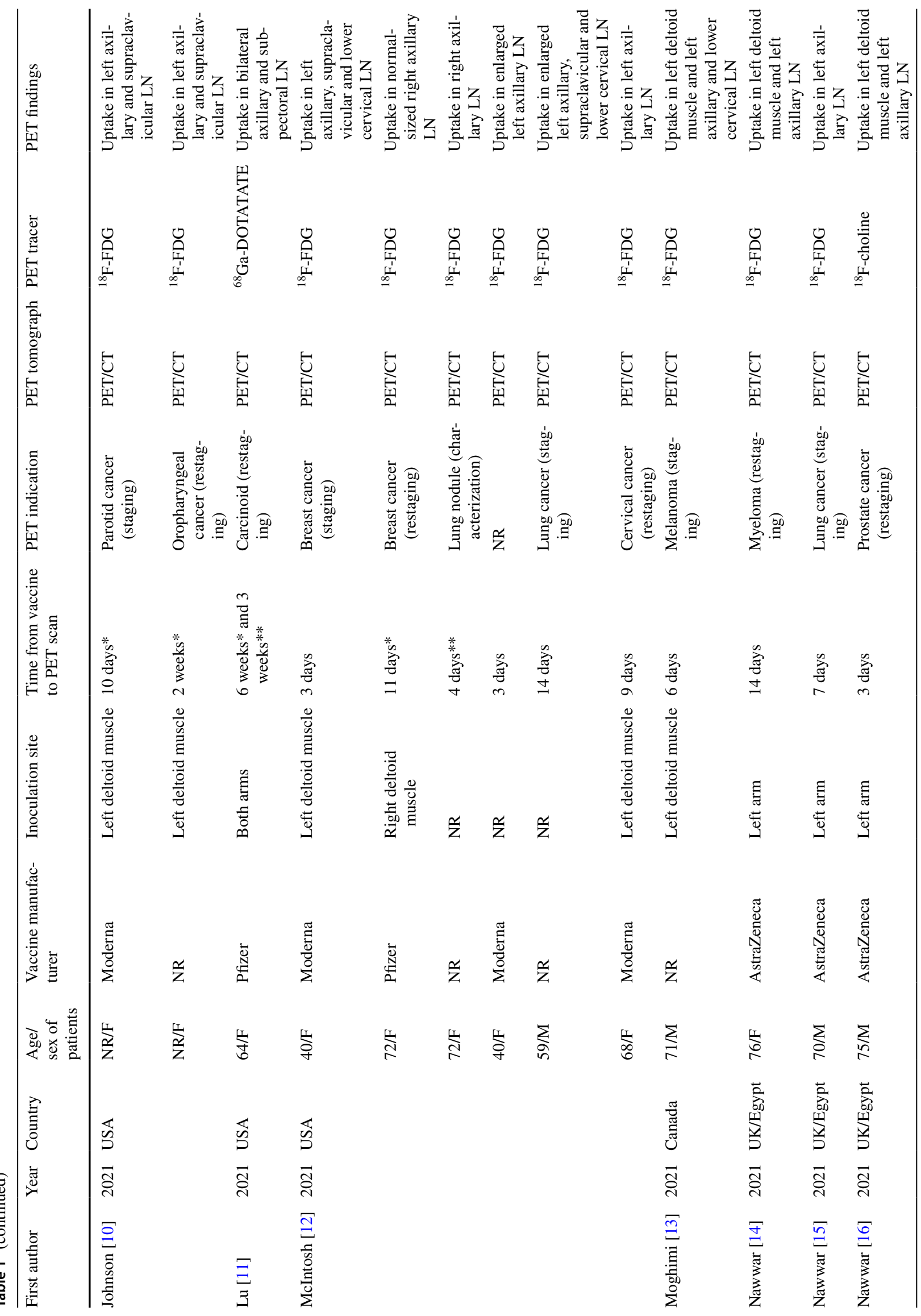




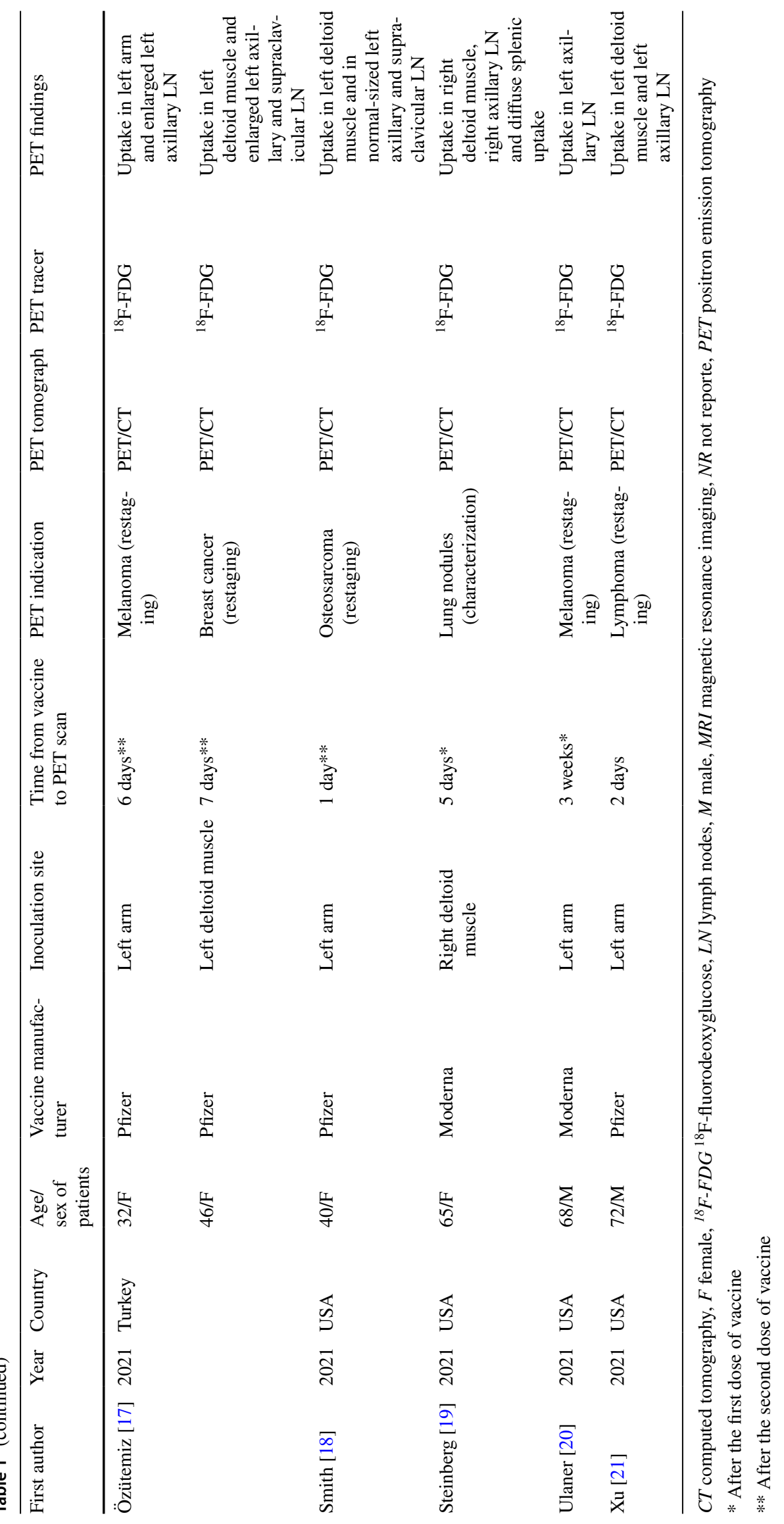


Table 2 Recent studies about the prevalence of COVID-19 vaccine-related lymphadenopathies on ${ }^{18}$ F-FDG PET/CT (source: PubMed/MEDLINE; last search date: 22 April 2021)

\begin{tabular}{lllllllll}
\hline First author & Year & Country & $\begin{array}{l}\text { No. of COVID-19 } \\
\text { vaccine recipients }\end{array}$ & $\begin{array}{l}\text { mean age/ } \\
\text { male per- } \\
\text { centage }\end{array}$ & $\begin{array}{l}\text { Vaccine } \\
\text { manufac- } \\
\text { turer }\end{array}$ & $\begin{array}{l}\text { Overall prevalence } \\
\text { of HALN after } \\
\text { COVID-19 vac- } \\
\text { cination }\end{array}$ & $\begin{array}{l}\text { Prevalence of } \\
\text { HALN after first } \\
\text { dose of COVID-19 } \\
\text { vaccine }\end{array}$ & $\begin{array}{l}\text { Prevalence of } \\
\text { HALN after second } \\
\text { dose of COVID-19 } \\
\text { vaccine }\end{array}$ \\
\hline Bernstine [27] & 2021 & Israel & 650 & $68.9 \mathrm{y} / 46 \%$ & Pfizer & $25.8 \%$ & $14.5 \%$ & $43.3 \%$ \\
Cohen [28] & 2021 & Israel & 728 & $69.2 \mathrm{y} / 43 \%$ & Pfizer & $45.6 \%$ & $36.4 \%$ & $53.9 \%$ \\
\hline
\end{tabular}

$H A L N$ hypermetabolic axillary lymph nodes at ${ }^{18}$ F-FDG PET

cases, because functional abnormalities as revealed by ${ }^{18} \mathrm{~F}-$ FDG PET may precede morphological alterations detected by CT or MRI [24]. Similar to ${ }^{18}$ F-FDG, radiopharmaceutical uptake in reactive lymph nodes has been already widely described with PET using radiolabelled choline [25] or somatostatin analogues [26].

Moreover, increased ${ }^{18} \mathrm{~F}$-FDG uptake in hypermetabolic lymph nodes due to vaccine-related immune response has been already described in several patients who underwent different types of vaccinations beyond those against COVID19 [22], therefore this is not a significant novelty.

Furthermore, we should also take into account that a clear information about the prevalence of these PET findings in COVID-19 vaccine recipients cannot be obtained by using these case reports and small case series only, because these manuscripts are strongly affected by publication bias; in other words, positive results (presence of increased radiopharmaceutical uptake at PET with different radiotracers after vaccination) are more likely to be published compared to negative findings (absence of increased radiopharmaceutical uptake at PET with different radiotracers after vaccination).

Conversely, two interesting cohort studies from Israel demonstrated that the detection of hypermetabolic axillary lymph nodes at ${ }^{18} \mathrm{~F}$-FDG PET/CT is quite common after vaccination against COVID-19, mainly after the inoculation of the second dose of COVID-19 vaccine (Table 2) [27, 28]. However, accurate data reporting the time required after COVID-19 vaccination to allow for resolution of ${ }^{18} \mathrm{~F}$-FDG uptake in sites of vaccine-related immune response are currently lacking.

Notably, taking into account all the evidence-based data available so far, we cannot state that PET with ${ }^{18} \mathrm{~F}-\mathrm{FDG}$ or other radiopharmaceuticals are really able or may be used to detect COVID-19 vaccination sequelae as well as for COVID-19 [29, 30]. It could be interesting to perform a trial in the future for evaluating if the increased ${ }^{18} \mathrm{~F}$-FDG uptake associated with the vaccination could give some useful information on the immune response for vaccinated individuals (as example: duration of immunity) or showing different behaviours when using different types of vaccine.

To date, we can only state that, in a still unclear percentage of COVID-19 vaccine recipients, some radiopharmaceutical uptake patterns as those described in the available articles may be found and these may be due to vaccine-related immune response. These PET findings will likely increase in number in the next months due to the parallel increase of global immunization against COVID-19.

Nuclear medicine physicians should be (already) able to recognize the possible PET findings due to COVID-19 vaccination, in particular both hypermetabolic lymph nodes (mainly axillary) and ipsilateral increased radiopharmaceutical uptake in the deltoid muscle at ${ }^{18}$ F-FDG PET. Documenting vaccination history and vaccine injection location at the time of PET scan is (already) extremely useful for PET reporters to avoid false interpretation, useless further diagnostic examinations, unnecessary changes in management and additional patient anxiety and this should be valid for all (COVID-19 and beyond) vaccine recipients.

\section{Declarations}

Conflict of interest The authors declare that they have no financial or non-financial competing interests.

Ethical approval This article does not contain any studies with human participants or animals.

\section{References}

1. Tu W, Gierada DS, Joe BN (2021) COVID-19 vaccination-related lymphadenopathy: what to be aware of. Radiol Imaging Cancer 3(3):e210038. https://doi.org/10.1148/rycan.2021210038

2. Ahmed N, Muzaffar S, Binns C, Ilyas MW, Usmani S (2021) COVID-19 vaccination manifesting as incidental lymph nodal uptake on 18F-FDG PET/CT. Clin Nucl Med. https://doi.org/10. 1097/RLU.0000000000003635

3. Avner M, Orevi M, Caplan N, Popovtzer A, Lotem M, Cohen JE (2021) COVID-19 vaccine as a cause for unilateral lymphadenopathy detected by 18 F-FDG PET/CT in a patient affected by melanoma. Eur J Nucl Med Mol Imaging. https://doi.org/10.1007/ s00259-021-05278-3

4. Bauckneht M, Aloè T, Tagliabue E, Cittadini G, Guadagno A, Morbelli S, Barisione E (2021) Beyond Covid-19 vaccinationassociated pitfalls on [18F]Fluorodeoxyglucose (FDG) PET: a 
case of a concomitant sarcoidosis. Eur J Nucl Med Mol Imaging. https://doi.org/10.1007/s00259-021-05360-w

5. Brown AH, Shah S, Groves AM, Wan S, Malhotra A (2021) The challenge of staging breast cancer with PET/CT in the era of COVID vaccination. Clin Nucl Med. https://doi.org/10.1097/ RLU.0000000000003683

6. Doss M, Nakhoda SK, Li Y, Yu JQ (2021) COVID-19 vaccinerelated local FDG uptake. Clin Nucl Med. https://doi.org/10.1097/ RLU.0000000000003634

7. Eifer M, Eshet Y (2021) Imaging of COVID-19 vaccination at FDG PET/CT. Radiology. https://doi.org/10.1148/radiol.20202 10030

8. Finnegan J, Govender P, Torreggiani WC (2021) Re: unilateral axillary adenopathy in the setting of COVID-19 vaccine. Clin Imaging. https://doi.org/10.1016/j.clinimag.2021.02.045

9. Hanneman K, Iwanochko RM, Thavendiranathan P (2021) Evolution of lymphadenopathy at PET/MRI after COVID-19 vaccination. Radiology. https://doi.org/10.1148/radiol.2021210386

10. Johnson BJ, Van Abel K, Ma D, Johnson DR (2021) FDG avid axillary lymph nodes after COVID-19 vaccination. J Nucl Med. https://doi.org/10.2967/jnumed.121.262108

11. Lu Y (2021) DOTATATE - avid bilateral axilla and subpectoral lymphadenopathy induced from COVID-19 mRNA vaccination visualized on PET/CT. Clin Nucl Med. https://doi.org/10.1097/ RLU.0000000000003697

12. McIntosh LJ, Bankier AA, Vijayaraghavan GR, Licho R, Rosen MP (2021) COVID-19 vaccination-related uptake on FDG PET/ CT: an emerging dilemma and suggestions for management. AJR Am J Roentgenol. https://doi.org/10.2214/AJR.21.25728

13. Moghimi S, Wilson D, Martineau P (2021) FDG PET findings post-COVID vaccinations: signs of the times? Clin Nucl Med. https://doi.org/10.1097/RLU.0000000000003636

14. Nawwar AA, Searle J, Hagan I, Lyburn ID (2021) COVID-19 vaccination induced axillary nodal uptake on [18F]FDG PET/ CT. Eur J Nucl Med Mol Imaging. https://doi.org/10.1007/ s00259-021-05274-7

15. Nawwar AA, Searle J, Hopkins R, Lyburn ID (2021) Falsepositive axillary lymph nodes on FDG PET/CT resulting from COVID-19 immunization. Clin Nucl Med. https://doi.org/10. 1097/RLU.0000000000003657

16. Nawwar AA, Searle J, Singh R, Lyburn ID (2021) Oxford-AstraZeneca COVID-19 vaccination induced lymphadenopathy on [18F]Choline PET/CT-not only an FDG finding. Eur J Nucl Med Mol Imaging. https://doi.org/10.1007/s00259-021-05279-2

17. Özütemiz C, Krystosek LA, Church AL, Chauhan A, Ellermann JM, Domingo-Musibay E, Steinberger D (2021) Lymphadenopathy in COVID-19 vaccine recipients: diagnostic dilemma in oncology patients. Radiology. https://doi.org/10.1148/radiol.20212 10275

18. Smith MV, Yang M (2021) Reactive axillary lymphadenopathy to COVID-19 vaccination on F18-FDG PET/CT. J Nucl Med Technol. https://doi.org/10.2967/jnmt.121.262008

19. Steinberg J, Thomas A, Iravani A (2021) ${ }^{18} \mathrm{~F}$-fluorodeoxyglucose PET/CT findings in a systemic inflammatory response syndrome after COVID-19 vaccine. Lancet 397(10279):e9. https://doi.org/ 10.1016/S0140-6736(21)00464-5

20. Ulaner GA, Giuliano P (2021) 18F-FDG-avid lymph nodes after COVID-19 vaccination on 18F-FDG PET/CT. Clin Nucl Med 46(5):433-434. https://doi.org/10.1097/RLU.0000000000003633

21. Xu G, Lu Y (2021) COVID-19 mRNA vaccination-induced lymphadenopathy mimics lymphoma progression on FDG PET/CT. Clin Nucl Med 46(4):353-354. https://doi.org/10.1097/RLU. 0000000000003597

22. Katal S, Pouraryan A, Gholamrezanezhad A (2021) COVID19 vaccine is here: practical considerations for clinical imaging applications. Clin Imaging 76:38-41. https://doi.org/10.1016/j. clinimag.2021.01.023

23. González-Gómez S, Lizarazo DA, Romero J (2021) FDG PET/CT scan after vaccination in times of pandemic. Radiology. https:// doi.org/10.1148/radiol.2021210447

24. Treglia G (2019) Diagnostic performance of ${ }^{18}$ F-FDG PET/CT in infectious and inflammatory diseases according to published meta-analyses. Contrast Media Mol Imaging 2019:3018349

25. Oprea-Lager DE, Vincent AD, van Moorselaar RJ, Gerritsen WR, van den Eertwegh AJ, Eriksson J, Boellaard R, Hoekstra OS (2012) Dual-phase PET-CT to differentiate [18F]Fluoromethylcholine uptake in reactive and malignant lymph nodes in patients with prostate cancer. PLoS One 7(10):e48430

26. Treglia G, Castaldi P, Rindi G, Giordano A, Rufini V (2012) Diagnostic performance of gallium-68 somatostatin receptor PET and $\mathrm{PET} / \mathrm{CT}$ in patients with thoracic and gastroenteropancreatic neuroendocrine tumours: a meta-analysis. Endocrine 42(1):80-87. https://doi.org/10.1007/s12020-012-9631-1

27. Bernstine H, Priss M, Anati T, Turko O, Gorenberg M, Steinmetz AP, Groshar D (2021) Axillary lymph nodes hypermetabolism after BNT162b2 mRNA COVID-19 vaccination in cancer patients undergoing 18F-FDG PET/CT: a cohort study. Clin Nucl Med 46(5):396-401. https://doi.org/10.1097/RLU.0000000000003648

28. Cohen D, Krauthammer SH, Wolf I, Even-Sapir E (2021) Hypermetabolic lymphadenopathy following administration of BNT162b2 mRNA Covid-19 vaccine: incidence assessed by [18F] FDG PET-CT and relevance to study interpretation. Eur J Nucl Med Mol Imaging. https://doi.org/10.1007/s00259-021-05314-2

29. Treglia G (2020) The role of ${ }^{18}$ F-FDG PET for COVID-19 infection: myth versus reality. Clin Transl Imaging. https://doi.org/10. 1007/s40336-020-00367-z

30. Annunziata S, Delgado Bolton RC, Kamani CH, Prior JO, Albano D, Bertagna F, Treglia G (2020) Role of 2- $\left[{ }^{18} \mathrm{~F}\right] \mathrm{FDG}$ as a radiopharmaceutical for PET/CT in patients with COVID-19: a systematic review. Pharmaceuticals (Basel) 13(11):377. https://doi.org/ $10.3390 / \mathrm{ph} 13110377$

Publisher's Note Springer Nature remains neutral with regard to jurisdictional claims in published maps and institutional affiliations. 